\title{
New Oscillation Criteria for Second-Order Forced Quasilinear Functional Differential Equations
}

\author{
Mervan Pašić \\ Department of Mathematics, Faculty of Electrical Engineering and Computing, University of Zagreb, 10000 Zagreb, Croatia \\ Correspondence should be addressed to Mervan Pašić; mervan.pasic@gmail.com
}

Received 29 April 2013; Accepted 17 July 2013

Academic Editor: Milan Tvrdy

Copyright (C) 2013 Mervan Pašić. This is an open access article distributed under the Creative Commons Attribution License, which permits unrestricted use, distribution, and reproduction in any medium, provided the original work is properly cited.

\begin{abstract}
We establish some new interval oscillation criteria for a general class of second-order forced quasilinear functional differential equations with $\phi$-Laplacian operator and mixed nonlinearities. It especially includes the linear, the one-dimensional $p$-Laplacian, and the prescribed mean curvature quasilinear differential operators. It continues some recently published results on the oscillations of the second-order functional differential equations including functional arguments of delay, advanced, or delay-advanced types. The nonlinear terms are of superlinear or supersublinear (mixed) types. Consequences and examples are shown to illustrate the novelty and simplicity of our oscillation criteria.
\end{abstract}

\section{Introduction}

We study the oscillation of the following three kinds of second-order forced quasilinear functional differential equations of delay, advanced, and delay-advanced types:

$$
\begin{aligned}
& \left(r(t) \phi\left(x^{\prime}(t)\right)\right)^{\prime}+\sum_{i=1}^{n} r_{i}(t) f\left(x\left(h_{i}(t)\right)\right) \\
& \quad+\sum_{i=1}^{n} q_{i}(t)\left|x\left(h_{i}(t)\right)\right|^{\alpha_{i}} \operatorname{sgn} x\left(h_{i}(t)\right)=e(t),
\end{aligned}
$$

where $t \geq t_{0}>0$ and $r(t), r_{i}(t), q_{i}(t)$, and $e(t)$ are continuous functions on $\left[t_{0}, \infty\right)$, and $x=x(t), x \in C^{2}\left(\left(t_{0}, \infty\right), \mathbb{R}\right)$, is a classic solution of (1). A continuous function $x(t)$ is said to be nonoscillatory if there is a $T>t_{0}$ such that $x(t) \neq 0$ on $[T, \infty)$. Otherwise, $x(t)$ is said to be oscillatory. Equation (1) is called oscillatory if all of its classic solutions are oscillatory.

In the delay case $h_{i}(t):=\tau_{i}(t) \leq t, i \in\{1, \ldots, n\}$, and $r(t)$ is nondecreasing; in the advanced case $h_{i}(t):=\sigma_{i}(t) \geq$ $t, i \in\{1, \ldots, n\}$, and $r(t)$ is nonincreasing; in the delayadvanced case $h_{i}(t):=\tau_{i}(t) \leq t, i \in\{1, \ldots, m\}, h_{i}(t):=$ $\sigma_{i}(t) \geq t, i \in\{m+1, \ldots, n\}$, and $r(t) \equiv$ const. $>0$. The exponents $\left\{\alpha_{i}\right\}$ satisfy a superlinear or a supersublinear (mixed) condition. On the function $\phi: \mathbb{R} \rightarrow \mathbb{R}$ which appears in the first term of (1), we impose such conditions that the following three main classes of second-order differential operators are especially included: the linear $\left(r(t) x^{\prime}\right)^{\prime}$, the onedimensional $p$-Laplacian $\left(r(t)\left|x^{\prime}\right|^{p-1} x^{\prime}\right)^{\prime}$, and the prescribed mean curvature operator $\left(r(t) x^{\prime}\left(1+x^{\prime 2}\right)^{-1 / 2}\right)^{\prime}$. The function $f(u)$ satisfies a usual growth condition and the coefficients $r_{i}(t)$, and $q_{i}(t)$ are positive only on some intervals where $e(t)$ changes the sign.

Recently, Bai and Liu [1] have studied the oscillation of second-order delay differential equation:

$$
\begin{aligned}
& \left(r(t) x^{\prime}(t)\right)^{\prime}+\sum_{i=1}^{n} r_{i}(t) x\left(t-\tau_{i}\right) \\
& \quad+\sum_{i=1}^{n} q_{i}(t)\left|x\left(t-\tau_{i}\right)\right|^{\alpha_{i}} \operatorname{sgn} x\left(t-\tau_{i}\right)=e(t), \quad t \geq t_{0},
\end{aligned}
$$


where $\tau_{i} \geq 0$. In Murugadass et al. [2], authors have studied the oscillation of the second-order quasilinear delay differential equation:

$$
\begin{aligned}
& \left(r(t)\left(x^{\prime}(t)\right)^{p}\right)^{\prime}+q(t) x^{p}(t-\tau) \\
& \quad+\sum_{i=1}^{n} q_{i}(t)|x(t-\tau)|^{\alpha_{i}} \operatorname{sgn} x(t-\tau)=e(t), \quad t \geq t_{0},
\end{aligned}
$$

where $p$ and $\left\{\alpha_{i}\right\}$ are ratio of odd positive integers. Later, in Hassan et al. [3], authors consider the oscillation of the halflinear functional differential equation:

$$
\begin{aligned}
& \left(r(t)\left(x^{\prime}(t)\right)^{p}\right)^{\prime}+r_{0}(t) x^{p}\left(h_{0}(t)\right) \\
& \quad+\sum_{i=1}^{n} q_{i}(t)\left|x\left(h_{i}(t)\right)\right|^{\alpha_{i}} \operatorname{sgn} x\left(h_{i}(t)\right)=e(t), \quad t \geq t_{0},
\end{aligned}
$$

where the function $h_{i}=h_{i}(t)$ is positive continuous functions with $\lim _{t \rightarrow \infty} h_{i}(t)=\infty$. Moreover, in [1-3] some doubts concerning the proof of the main result of [4] are resolved. The well-known variational technique that uses the generalized Philos' results based on the so-called $H$-function has been used in [1, Theorem 2.2], [2, Theorems 2.3, and 2.6], and [3, Theorems 2.5, 2.6, and 2.7] (see also [5-11] and references therein). Since the second-order quasilinear differential operator $\left(r(t) \phi\left(x^{\prime}(t)\right)\right)^{\prime}$ usually causes some difficulties in many problems, the application of previous method on (1) is stated here as an open problem. In contrast to the preceding, we use a combination of the Riccati classic transformation, a blow-up argument, and a comparison pointwise principle recently established in $[12,13]$ but for differential equations without functional arguments. It seems that our criteria are slightly simpler to be verified, which is discussed on some examples given in the next section.

Among some recently published papers on the oscillation of quasilinear functional differential equations with both delay and advanced terms, we point out an oscillation criterion by Zafer [5] obtained for equation

$$
\begin{aligned}
& \left(\left|x^{\prime}(t)\right|^{p^{-1}} x^{\prime}(t)\right)^{\prime}+q_{1}(t)|x(\tau(t))|^{\beta-1} x(\tau(t)) \\
& \quad+q_{2}(t)|x(\sigma(t))|^{\gamma-1} x(\sigma(t))=e(t), \quad t \geq 0,
\end{aligned}
$$

where $p>0, \beta \geq p$, and $\gamma \geq p$. On an interesting example in [5], the author established the oscillations provided at least one of constants appearing in the coefficients is sufficiently large (see also [6] for $p=1$ ), which is presented here in the next section as a particular case of our main results.

On the properties of some classes of the one-dimensional quasilinear differential equations with $\phi$-Laplacian operators we refer reader to [14-20] and the references therein. About the applications of second-order functional differential equations in the mathematical description of certain phenomena in physics, technics, and biology (oscillation in a vacuum tube; interaction of an oscillator with an energy source; coupled oscillators in electronics, chemistry, and ecology; relativistic motion of a mass in a central field; ship course stabilization; moving of the tip of a growing plant; etc.), we suggest reading Kolmanovskii and Myshkis book [21].

\section{Main Results and Examples}

Let $\phi=\phi(v)$ be a function which appears in the first term of (1) such that

$\phi \in C^{1}(\mathbb{R}, \mathbb{R}), \quad \phi$ is odd and increasing function on $\mathbb{R}$,

$$
\phi(v) v \geq|\phi(v)|^{(p+1) / p} \quad \forall v \in \mathbb{R} \text { and some } p>0 .
$$

These two assumptions are fulfilled, respectively, in the linear case $\left(r(t) x^{\prime}(t)\right)^{\prime}$, where $\phi(v) \equiv v$ and $p=$ 1 ; in the one-dimensional $p$-Laplacian quasilinear case $\left(r(t)\left|x^{\prime}(t)\right|^{p-1} x^{\prime}(t)\right)^{\prime}$, where $\phi(v) \equiv|v|^{p-1} v$ and $p>0$; and in the mean prescribed curvature quasilinear case

$$
\left[r(t) x^{\prime}(t)\left(1+x^{\prime 2}(t)\right)^{-1 / 2}\right]^{\prime}
$$

where $\phi(v)=v\left(1+v^{2}\right)^{-1 / 2}$ and $p=1$. The importance of the prescribed mean curvature quasilinear differential operators lies in the capillarity type problems in fluid mechanics, fluxlimited diffusion phenomena, and prescribed mean curvature problems; see for instance [14, 22, 23].

The function $f=f(u)$ which appears in the second term of (1) satisfies

$$
\begin{aligned}
& f(u) \text { is odd function on } \mathbb{R}, \\
& \frac{f(u)}{u^{p}} \geq K>0 \quad \forall u>0 \text {, and some } K \in \mathbb{R},
\end{aligned}
$$

where $p>0$ is from assumption (7).

In the first two theorems below, the exponents $\left\{\alpha_{i}\right\}$ satisfy

$$
\begin{aligned}
& \alpha_{1} \geq \cdots \geq \alpha_{m}>p>\alpha_{m+1} \geq \cdots \geq \alpha_{n}>0, \quad m \in \mathbb{N}, \\
& \qquad \alpha_{i}>\alpha_{i+1}, \quad i \in\{1, \ldots, n-1\} ; \\
& \text { there exists }(n+1) \text {-tuple }\left(\eta_{0}, \eta_{1}, \ldots, \eta_{n}\right) \\
& \text { such that } 0<\eta_{i}<1, \quad \sum_{i=1}^{n} \eta_{i}<1, \quad \eta_{0}=1-\sum_{i=1}^{n} \eta_{i}, \\
& \qquad \sum_{i=1}^{n} \alpha_{i} \eta_{i}=p
\end{aligned}
$$

where $p$ is from assumption (7). For instance if $n=2, \alpha_{1}=$ $5 / 2, p=1$, and $\alpha_{2}=1 / 2$, then $\eta_{0}=\eta_{1}=\eta_{2}=1 / 3$. On assumption (10) see for instance [9, Lemma 1].

Unlike recently published oscillation criteria for the linear and half-linear second-order forced functional differential 
equations, our oscillation criterion is only based on the following elementary integral inequality:

$$
\frac{1}{2 \pi_{p}} \int_{a_{j}}^{b_{j}} \min \left\{\frac{p}{\left(\lambda_{j} r(t)\right)^{1 / p}}, \lambda_{j}\left(R_{j}(t)+Q_{j}(t)\right)\right\} d t \geq 1,
$$$$
j \in\{1,2\} \text {, }
$$

where $a_{1}<b_{1} \leq a_{2}<b_{2}, p$ is from (7), $\pi_{p}=(p /(p+$ 1)) $\pi / \sin (p \pi /(p+1)), \lambda_{j}>0$, and functions $Q_{j}(t)$, and $R_{j}(t)$ are explicitly expressed by the coefficients of (1) just like it is done in the next three main results.

Theorem 1 (delay equation). One assumes (6), (7), (9), and (10). Let $r(t)$ be a nondecreasing positive function on $\left[t_{0}, \infty\right)$. Let $h_{i}(t)=\tau_{i}(t) \leq t$ on $\left[t_{0}, \infty\right)$ and $\lim _{t \rightarrow \infty} \tau_{i}(t)=\infty, i \in$ $\{1,2, \ldots, n\}$. Let for every $T \geq t_{0}$ there exist $a_{1}, b_{1}, a_{2}, b_{2}, T \leq$ $a_{1}<b_{1} \leq \tau_{\min }\left(a_{2}\right) \leq a_{2}<b_{2}$ such that

$$
\begin{gathered}
r_{i}(t) \geq 0, \quad q_{i}(t) \geq 0 \\
\text { on }\left[\tau_{\min }\left(a_{1}\right), b_{1}\right] \cup\left[\tau_{\min }\left(a_{2}\right), b_{2}\right], \\
e(t) \leq 0 \quad \text { on }\left[\tau_{\min }\left(a_{1}\right), b_{1}\right], \\
e(t) \geq 0 \quad \text { on }\left[\tau_{\min }\left(a_{2}\right), b_{2}\right],
\end{gathered}
$$

where $\tau_{\min }(t)=\min \left\{\tau_{1}(t), \tau_{2}(t), \ldots, \tau_{n}(t)\right\}$. Equation (1) is oscillatory provided there are two real parameters $\lambda_{1}, \lambda_{2}>0$ such that (11) is fulfilled, where

$$
R_{j}(t)=K \sum_{i=1}^{n} r_{i}(t)\left(\frac{\tau_{i}(t)-\tau_{i}\left(a_{j}\right)}{t-\tau_{i}\left(a_{j}\right)}\right)^{p},
$$

$$
\begin{aligned}
& Q_{j}(t) \\
& \quad=\left(\eta_{0}^{-1}|e(t)|\right)^{\eta_{0}} \prod_{i=1}^{n}\left(\eta_{i}^{-1} q_{i}(t)\right)^{\eta_{i}} \prod_{i=1}^{n}\left(\frac{\tau_{i}(t)-\tau_{i}\left(a_{j}\right)}{t-\tau_{i}\left(a_{j}\right)}\right)^{\alpha_{i} \eta_{i}},
\end{aligned}
$$

for $t \in\left[a_{j}, b_{j}\right], j \in\{1,2\}$, and positive constants $p, K$, $\eta_{i}$ appearing, respectively, in (7), (9), and (10).

As we can see in (13), the forcing term $e(t)$ can be an oscillatory function. The main property of any interval oscillation criterion (see [8]) is that the coefficients of the considered equation do not satisfy some conditions on the whole $\left[t_{0}, \infty\right)$ than only on intervals $\left[a_{1}, b_{1}\right] \cup\left[a_{2}, b_{2}\right]$. The main consequence of Theorem 1 is the following oscillation criterion for (1) with $r(t) \equiv 1$ and $r_{i}(t) \equiv 0, i \in\{1,2, \ldots, n\}$.

Corollary 2. One assumes (6), (7), (10), (12), and (13). Then equation

$$
\left(\phi\left(x^{\prime}(t)\right)\right)^{\prime}+\sum_{i=1}^{n} q_{i}(t)\left|x\left(\tau_{i}(t)\right)\right|^{\alpha_{i}} \operatorname{sgn} x\left(\tau_{i}(t)\right)=e(t)
$$

is oscillatory provided

$$
\begin{array}{r}
\frac{p^{p /(p+1)}}{2 \pi_{p}} \int_{a_{j}}^{b_{j}} Q_{j}(t) d t \geq\left(\max _{t \in\left[a_{j}, b_{j}\right]} Q_{j}(t)\right)^{p /(p+1)}>0, \\
j \in\{1,2\},
\end{array}
$$

where $Q_{j}(t)$ is defined in (15).

In particular for $p=1$, previous corollary takes the following form.

Corollary 3. Let (10) hold with $p=1, \tau_{i}(t) \leq t$ on $\left[t_{0}, \infty\right)$, and $\lim _{t \rightarrow \infty} \tau_{i}(t)=\infty, i \in\{1,2, \ldots, n\}$. Let for every $T \geq$ $t_{0}$ there exist $a_{1}, b_{1}, a_{2}, b_{2}, T \leq a_{1}<b_{1} \leq \tau_{\min }\left(a_{2}\right) \leq a_{2}<$ $b_{2}$ such that assumptions (12) and (13) hold with $r_{i}(t) \equiv 0$. Equation

$$
x^{\prime \prime}(t)+\sum_{i=1}^{n} q_{i}(t)\left|x\left(\tau_{i}(t)\right)\right|^{\alpha_{i}} \operatorname{sgn} x\left(\tau_{i}(t)\right)=e(t)
$$

is oscillatory provided

$$
\frac{1}{\pi} \int_{a_{j}}^{b_{j}} Q_{j}(t) d t \geq \sqrt{\max _{t \in\left[a_{j}, b_{j}\right]} Q_{j}(t)}>0, \quad j \in\{1,2\},
$$

where $Q_{j}(t)$ is defined in $(15)$.

In the next examples and remarks, we discuss the application of oscillation criteria from Corollary 3 and [1, Theorem 2.2] on the following equation:

$$
\begin{aligned}
x^{\prime \prime} & +m_{1} \sin (t)\left|x\left(\tau_{1}(t)\right)\right|^{\alpha_{1}} \operatorname{sgn} x\left(\tau_{1}(t)\right) \\
& +m_{2} \cos (t)\left|x\left(\tau_{2}(t)\right)\right|^{\alpha_{2}} \operatorname{sgn} x\left(\tau_{2}(t)\right)=-e_{0} \cos (2 t),
\end{aligned}
$$

where $\alpha_{1}>1>\alpha_{2}>0, \tau_{1}(t)=\tau_{2}(t)=t-\pi / 8$, and $m_{1}, m_{2}$, and $e_{0}$ are positive constants.

Example 4. As a consequence of Corollary 3, we show in this example that (20) is oscillatory provided the constants $m_{1}, m_{2}$, and $e_{0}$ satisfy the following simple inequality:

$$
\frac{I_{j}}{\pi} \sqrt{\eta e_{0}^{\eta_{0}} m_{1}^{\eta_{1}} m_{2}^{\eta_{2}}} \geq 1, \quad j \in\{1,2\},
$$

where $\eta_{i}$ satisfy $(10), \eta=\prod_{i=0}^{2} \eta_{i}^{-\eta_{i}}$, and $I_{1}, I_{2}>0$ are two real numbers defined by

$$
\begin{aligned}
I_{1} & =\int_{\pi / 8}^{\pi / 4} W(t) \frac{t-\pi / 8}{t} d t, \\
I_{2} & =\int_{3 \pi / 8}^{\pi / 2} W(t) \frac{t-3 \pi / 8}{t-\pi / 4} d t, \\
W(t) & =|\cos 2 t|^{\eta_{0}}|\sin t|^{\eta_{1}}|\cos t|^{\eta_{2}} .
\end{aligned}
$$

Indeed, let $\pi_{p}=\pi / 2, \tau_{\min }(t)=\tau_{1}(t)=\tau_{2}(t)=t-$ $\pi / 8,\left[a_{1}, b_{1}\right]=[\pi / 8+2 k \pi, \pi / 4+2 k \pi],\left[a_{2}, b_{2}\right]=[3 \pi / 8+$ 
$2 k \pi, \pi / 2+2 k \pi], k \in \mathbb{N}, q_{1}(t)=\sin (t), q_{2}(t)=\cos (t)$, and $e(t)=-e_{0} \cos (2 t)$. Firstly, it is elementary to check that the required inequalities (12) and (13) are satisfied. Next, we prove that required inequality (19) immediately follows from assumption (21). On the first hand, we estimate from above the term on the right hand side in (19) using that $\alpha_{1} \eta_{1}+\alpha_{2} \eta_{2}=$ 1:

$$
\begin{array}{r}
Q_{j}(t)=\eta e_{0}^{\eta_{0}} m_{1}^{\eta_{1}} m_{2}^{\eta_{2}} W(t) \frac{t-a_{j}}{t-a_{j}+\pi / 8} \leq \eta e_{0}^{\eta_{0}} m_{1}^{\eta_{1}} m_{2}^{\eta_{2}}, \\
t \in\left[a_{j}, b_{j}\right],
\end{array}
$$

that is

$$
0<\sqrt{\max _{t \in\left[a_{j}, b_{j}\right]} Q_{j}(t)} \leq \sqrt{\eta e_{0}^{\eta_{0}} m_{1}^{\eta_{1}} m_{2}^{\eta_{2}}}, \quad j \in\{1,2\} .
$$

On the other hand, we calculate the integral on the left hand side in (19):

$$
\begin{aligned}
& \int_{a_{1}}^{b_{1}} Q_{1}(t) d t \\
& \quad=\eta e_{0}^{\eta_{0}} m_{1}^{\eta_{1}} m_{2}^{\eta_{2}} \int_{\pi / 8+2 k \pi}^{\pi / 4+2 k \pi} W(t) \frac{t-(\pi / 8+2 k \pi)}{t-(\pi / 8+2 k \pi)+\pi / 8} d t \\
& \quad=\eta e_{0}^{\eta_{0}} m_{1}^{\eta_{1}} m_{2}^{\eta_{2}} I_{1}, \\
& \int_{a_{2}}^{b_{2}} Q_{2}(t) d t \\
& \quad=\eta e_{0}^{\eta_{0}} m_{1}^{\eta_{1}} m_{2}^{\eta_{2}} \int_{3 \pi / 8+2 k \pi}^{\pi / 2+2 k \pi} W(t) \frac{t-(3 \pi / 8+2 k \pi)}{t-(3 \pi / 8+2 k \pi)+\pi / 8} d t \\
& \quad=\eta e_{0}^{\eta_{0}} m_{1}^{\eta_{1}} m_{2}^{\eta_{2}} I_{2} .
\end{aligned}
$$

Now, from previous two equalities, and inequalities (21) and (24), we conclude

$$
\begin{aligned}
& \frac{1}{\pi} \int_{a_{j}}^{b_{j}} Q_{j}(t) d t=\frac{I_{j}}{\pi} \eta e_{0}^{\eta_{0}} m_{1}^{\eta_{1}} m_{2}^{\eta_{2}} \\
& \quad \geq \sqrt{\eta e_{0}^{\eta_{0}} m_{1}^{\eta_{1}} m_{2}^{\eta_{2}}} \geq \sqrt{\max _{t \in\left[a_{j}, b_{j}\right]} Q_{j}(t)}>0 .
\end{aligned}
$$

Thus, assumption (21) proves desired inequality (19), and thus, Corollary 2 verifies that (20) is oscillatory provided (21) holds.

Remark 5. One way to obtain the inequality (21) is to suppose, for instance, that at least one of the constants $m_{1}, m_{2}$, and $e_{0}$ is large enough.

Remark 6. We can consider the slightly more general equation than (20):

$$
\begin{aligned}
& \left(\phi\left(x^{\prime}(t)\right)\right)^{\prime}+m_{1} \sin (t)\left|x\left(t-\tau_{1}\right)\right|^{\alpha_{1}} \operatorname{sgn} x\left(t-\tau_{1}\right) \\
& \quad+m_{2} \cos (t)\left|x\left(t-\tau_{2}\right)\right|^{\alpha_{2}} \operatorname{sgn} x\left(t-\tau_{2}\right)=-e_{0} \cos (2 t),
\end{aligned}
$$

where $\tau_{1}=\tau_{2}=\pi / 8$ and $\phi=\phi(v)$ satisfy (6) and (7), and $\alpha_{1}>p>\alpha_{2}>0$. In a similar way as in Example 4, one can show that (27) is oscillatory provided at least one of the constants $m_{1}, m_{2}$, and $e_{0}$ is large enough.

Example 7. In this example we present an application of [1, Theorem 2.2] for getting another condition on the constants $m_{1}, m_{2}$, and $e_{0}$ for oscillation of (20). Let $\tau_{1}=$ $\tau_{2}=\pi / 8$,

$$
\begin{aligned}
& {\left[a_{1}, c_{1}\right]=\left[\frac{\pi}{8}+2 k \pi, \frac{3 \pi}{16}+2 k \pi\right],} \\
& {\left[c_{1}, b_{1}\right]=\left[\frac{3 \pi}{16}+2 k \pi, \frac{\pi}{4}+2 k \pi\right],} \\
& {\left[a_{2}, c_{2}\right]=\left[\frac{3 \pi}{8}+2 k \pi, \frac{7 \pi}{16}+2 k \pi\right],} \\
& {\left[c_{2}, b_{2}\right]=\left[\frac{7 \pi}{16}+2 k \pi, \frac{\pi}{2}+2 k \pi\right],}
\end{aligned}
$$

$q_{1}(t)=m_{1} \sin (t), q_{2}(t)=m_{2} \cos (t)$, and $e(t)=-e_{0} \cos (2 t)$. It is clear that $q_{i}(t) \geq 0$ on $\left[a_{1}-\tau_{i}, b_{1}\right] \cup\left[a_{2}-\tau_{i}, b_{2}\right], i=$ $1,2, e(t) \leq 0$ on $\left[a_{1}-\tau_{i}, b_{1}\right]$, and $e(t) \geq 0$ on $\left[a_{2}-\right.$ $\left.\tau_{i}, b_{2}\right], i=1,2$. Therefore we may apply [1, Theorem 2.2] on (20) provided the following inequality holds:

$$
\begin{array}{r}
\frac{1}{H_{j}\left(c_{j}, a_{j}\right)} \int_{a_{j}}^{c_{j}}\left(Q_{j}(t) H_{j}\left(t, a_{j}\right)-1\right) d t \\
+\frac{1}{H_{j}\left(b_{j}, c_{j}\right)} \int_{c_{j}}^{b_{j}}\left(Q_{j}(t) H_{j}\left(b_{j}, t\right)-1\right) d t>0, \\
\quad j \in\{1,2\} .
\end{array}
$$

Now, if we put $H_{1}(t, s)=H_{2}(t, s)=(t-s)^{2}$ and $h_{j, 1}$ $(t, s)=h_{j, 2}(t, s)=1$ for $t>s$, then previous inequality takes the following concrete form:

$$
\begin{aligned}
& \int_{\pi / 8+2 k \pi}^{3 \pi / 16+2 k \pi} Q_{1}(t)\left(t-\frac{\pi}{8}-2 k \pi\right)^{2} d t \\
& +\int_{3 \pi / 16+2 k \pi}^{\pi / 4+2 k \pi} Q_{1}(t)\left(t-\frac{\pi}{4}-2 k \pi\right)^{2} d t>\frac{\pi}{8}, \\
& \int_{3 \pi / 8+2 k \pi}^{7 \pi / 16+2 k \pi} Q_{2}(t)\left(t-\frac{3 \pi}{8}-2 k \pi\right)^{2} d t \\
& +\int_{7 \pi / 16+2 k \pi}^{\pi / 2+2 k \pi} Q_{2}(t)\left(t-\frac{\pi}{2}-2 k \pi\right)^{2} d t>\frac{\pi}{8} .
\end{aligned}
$$

Similarly as in Example 4, we can calculate previous integrals and conclude by [1, Theorem 2.2] that (20) is oscillatory provided the following inequalities hold:

$$
\begin{aligned}
& \eta e_{0}^{\eta_{0}} m_{1}^{\eta_{1}} m_{2}^{\eta_{2}}\left(I_{11}+I_{12}\right)>\frac{\pi}{8}, \\
& \eta e_{0}^{\eta_{0}} m_{1}^{\eta_{1}} m_{2}^{\eta_{2}}\left(I_{21}+I_{22}\right)>\frac{\pi}{8},
\end{aligned}
$$


where

$$
\begin{aligned}
& I_{11}=\int_{\pi / 8}^{3 \pi / 16} W(t) \frac{(t-\pi / 8)^{3}}{t} d t, \\
& I_{12}=\int_{3 \pi / 16}^{\pi / 4} W(t) \frac{(t-\pi / 8)(t-\pi / 4)^{2}}{t} d t, \\
& I_{21}=\int_{3 \pi / 8}^{7 \pi / 16} W(t) \frac{(t-3 \pi / 8)^{3}}{t-\pi / 4} d t, \\
& I_{22}=\int_{7 \pi / 16}^{\pi / 2} W(t) \frac{(t-3 \pi / 8)(t-\pi / 2)^{2}}{t-\pi / 4} d t,
\end{aligned}
$$

and $W(t)=|\cos 2 t|^{\eta_{0}}|\sin t|^{\eta_{1}}|\cos t|^{\eta_{2}}$.

We leave to the reader to compare and verify which one of the two inequalities (21) and (31) is simpler to be verified. Also, similar to the previous example, it is possible to get corresponding inequalities analogously to (31) for other criteria published in the papers cited in the references.

Remark 8. In [1, Section 3] the authors give an application of [1, Theorem 2.2] to (20) where $\tau_{1}(t)=t-\tau_{1}, \tau_{2}(t)=t-$ $\tau_{2}, \tau_{1}=\pi / 8$, and $\tau_{2}=\pi / 4$. However, the following choice (see $[1$, Section 3])

$$
\begin{gathered}
{\left[a_{1}, c_{1}\right]=\left[2 k \pi, \frac{\pi}{8}+2 k \pi\right],} \\
{\left[c_{1}, b_{1}\right]=\left[\frac{\pi}{8}+2 k \pi, \frac{\pi}{4}+2 k \pi\right],} \\
{\left[a_{2}, c_{2}\right]=\left[\frac{\pi}{4}+2 k \pi, \frac{3 \pi}{8}+2 k \pi\right],} \\
{\left[c_{2}, b_{2}\right]=\left[\frac{3 \pi}{8}+2 k \pi, \frac{\pi}{2}+2 k \pi\right],}
\end{gathered}
$$

is not correct since the desired conditions $q_{i}(t) \geq 0$ on $\left[a_{1}-\right.$ $\left.\tau_{i}, b_{1}\right] \cup\left[a_{2}-\tau_{i}, b_{2}\right], i=1,2$ are not fulfilled. Hence, we suggest reader to use the intervals proposed in the previous example.

Open Question 9. The well-known variational technique based on the generalized Philos' $H$-function has been used in [1-11] to obtain some oscillation criteria for (1), where the second-order quasilinear differential operator $\left(r(t) \phi\left(x^{\prime}(t)\right)\right)^{\prime}$ is linear or half-linear. Is it possible to use this technique in the case of any function $\phi(v)$ satisfying general conditions (6) and (7)?

Theorem 10 (advanced equation). Under assumptions (6), (7), (9), and (10), let $r(t)$ be a nonincreasing positive function on $\left[t_{0}, \infty\right)$ and $h_{i}(t)=\sigma_{i}(t) \geq t$ on $\left[t_{0}, \infty\right), i \in\{1,2, \ldots, n\}$. Let for every $T \geq t_{0}$ there exist numbers $a_{1}, b_{1}, a_{2}, b_{2}$, such that $T \leq a_{1}<b_{1} \leq \sigma_{\max }\left(b_{1}\right) \leq a_{2}<b_{2}$ and

$$
\begin{gathered}
r_{i}(t) \geq 0, \quad q_{i}(t) \geq 0 \\
\text { on }\left[a_{1}, \sigma_{\max }\left(b_{1}\right)\right] \cup\left[a_{2}, \sigma_{\max }\left(b_{2}\right)\right], \\
e(t) \leq 0 \text { on }\left[a_{1}, \sigma_{\max }\left(b_{1}\right)\right], \\
e(t) \geq 0 \text { on }\left[a_{2}, \sigma_{\max }\left(b_{2}\right)\right],
\end{gathered}
$$

where $\sigma_{\max }(t)=\max \left\{\sigma_{1}(t), \ldots, \sigma_{n}(t)\right\}$. Equation (1) is oscillatory provided there are two real parameters $\lambda_{1}, \lambda_{2}>0$ such that (11) is fulfilled, where

$$
R_{j}(t)=K \sum_{i=1}^{n} r_{i}(t)\left(\frac{\sigma_{i}\left(b_{j}\right)-\sigma_{i}(t)}{\sigma_{i}\left(b_{j}\right)-t}\right)^{p}
$$

$Q_{j}(t)$

$$
=\left(\eta_{0}^{-1}|e(t)|\right)^{\eta_{0}} \prod_{i=1}^{n}\left(\eta_{i}^{-1} q_{i}(t)\right)^{\eta_{i}} \prod_{i=1}^{n}\left(\frac{\sigma_{i}\left(b_{j}\right)-\sigma_{i}(t)}{\sigma_{i}\left(b_{j}\right)-t}\right)^{\alpha_{i} \eta_{i}},
$$

for $t \in\left[a_{j}, b_{j}\right], j \in\{1,2\}$, and positive constants $p, K$, and $\eta_{i}$ appearing, respectively, in (7), (9), and (10).

Now, analogously with (16), we consider the oscillation of the advanced equation:

$$
\left(\phi\left(x^{\prime}(t)\right)\right)^{\prime}+\sum_{i=1}^{n} q_{i}(t)\left|x\left(\sigma_{i}(t)\right)\right|^{\alpha_{i}} \operatorname{sgn} x\left(\sigma_{i}(t)\right)=e(t) .
$$

As a consequence of Theorem 10, we have the following criterion.

Corollary 11. One assumes (6), (7), (10), and (34). Then (37) is oscillatory provided condition (17) is satisfied, where $Q_{j}(t)$ is defined in (36).

As the third case, we consider second-order functional differential equations with both delay and advanced arguments.

Theorem 12 (delay-advanced equation). One assumes (6), (7), and (9), $\alpha_{i}>p$ for $i \in\{1,2, \ldots, n\}$, and $m \in \mathbb{N}, 1<$ $m<n$. Let $r(t) \equiv$ const $>0$ on $\left[t_{0}, \infty\right), h_{i}(t)=\tau_{i}(t) \leq$ $t$ for $i \in\{1, \ldots, m\}$, and $h_{i}(t)=\sigma_{i}(t) \geq t$ for $i \in\{m+$ $1, \ldots, n\}$ on $\left[t_{0}, \infty\right)$. Let $r_{i}(t), q_{i}(t)$, and $e(t)$ satisfy $(12)-(13)$ for $i \in\{1, \ldots, m\}$ and (34) for $i \in\{m+1, \ldots, n\}$. Equation (1) is oscillatory provided there are two real parameters $\lambda_{1}, \lambda_{2}>$ 0 such that (11) is fulfilled, where

$$
\begin{aligned}
R_{j}(t)= & K \sum_{i=1}^{m} r_{i}(t)\left(\frac{\tau_{i}(t)-\tau_{i}\left(a_{j}\right)}{t-\tau_{i}\left(a_{j}\right)}\right)^{p} \\
& +K \sum_{i=m+1}^{n} r_{i}(t)\left(\frac{\sigma_{i}\left(b_{j}\right)-\sigma_{i}(t)}{\sigma_{i}\left(b_{j}\right)-t}\right)^{p},
\end{aligned}
$$




$$
\begin{aligned}
Q_{j}(t)= & \sum_{i=1}^{m} \frac{\alpha_{i}}{p} q_{i}^{p / \alpha_{i}}(t)\left(\frac{p|e(t)|}{2\left(\alpha_{i}-p\right) m}\right)^{\left(\alpha_{i}-p\right) / \alpha_{i}} \\
& \times\left(\frac{\tau_{i}(t)-\tau_{i}\left(a_{j}\right)}{t-\tau_{i}\left(a_{j}\right)}\right)^{p} \\
+ & \sum_{i=m+1}^{n} \frac{\alpha_{i}}{p} q_{i}^{p / \alpha_{i}}(t)\left(\frac{p|e(t)|}{2\left(\alpha_{i}-p\right)(n-m-1)}\right)^{\left(\alpha_{i}-p\right) / \alpha_{i}} \\
& \times\left(\frac{\sigma_{i}\left(b_{j}\right)-\sigma_{i}(t)}{\sigma_{i}\left(b_{j}\right)-t}\right)^{p},
\end{aligned}
$$

for $t \in\left[a_{j}, b_{j}\right], j \in\{1,2\}$, and positive constants $p, K$ appearing, respectively, in (7) and (9).

Analogously with (16) and (37), we consider the oscillation of delay-advanced equation:

$$
\begin{gathered}
\left(\phi\left(x^{\prime}(t)\right)\right)^{\prime}+\sum_{i=1}^{m} q_{i}(t)\left|x\left(\tau_{i}(t)\right)\right|^{\alpha_{i}} \operatorname{sgn} x\left(\tau_{i}(t)\right) \\
+\sum_{i=m+1}^{n} q_{i}(t)\left|x\left(\sigma_{i}(t)\right)\right|^{\alpha_{i}} \operatorname{sgn} x\left(\sigma_{i}(t)\right)=e(t) .
\end{gathered}
$$

As a consequence of Theorem 12 we derive the following oscillation criterion for (40).

Corollary 13. Let all assumptions of Theorem 12 hold with respect to $r_{i}(t) \equiv 0$ and $f(u) \equiv 0$. Equation (40) is oscillatory provided condition (17) is satisfied, where $Q_{j}(t)$ is defined in (39).

In Zafer [5] (see also [6]) the author has illustrated its main oscillation result on the following example of the second-order functional differential equations with delay and advanced arguments. Since $|x|^{\alpha-1} x=|x|^{\alpha} \operatorname{sgn} x$, it can be rewritten in the form

$$
\begin{aligned}
& \left(\left|x^{\prime}(t)\right|^{p-1} x^{\prime}(t)\right)^{\prime}+m_{1} \sin (t)|x(\tau(t))|^{\alpha_{1}} \operatorname{sgn} x(\tau(t)) \\
& \quad+m_{2} \cos (t)|x(\sigma(t))|^{\alpha_{2}} \operatorname{sgn} x(\sigma(t))=-e_{0} \cos (2 t),
\end{aligned}
$$

where $m_{1}, m_{2}$, and $e_{0}$ are positive constants; $\tau(t)=t-\pi / 5$; $\sigma(t)=t+\pi / 20$; and $\alpha_{1}, \alpha_{2}>0$. It has been proved that previous equation is oscillatory provided at least one of the constants $m_{1}, m_{2}$, and $e_{0}$ is large enough. Here according to Corollary 13 we can repeat this interesting conclusion for slightly complicated equation but with $\alpha_{1}, \alpha_{2}>1$.

Example 14. We consider the following $\phi$-Laplacian functional differential equation with delay and advanced arguments:

$$
\begin{aligned}
& \left(\phi\left(x^{\prime}(t)\right)\right)^{\prime}+m_{1} \sin (t)|x(\tau(t))|^{\alpha_{1}} \operatorname{sgn} x(\tau(t)) \\
& \quad+m_{2} \cos (t)|x(\sigma(t))|^{\alpha_{2}} \operatorname{sgn} x(\sigma(t))=-e_{0} \cos (2 t),
\end{aligned}
$$

where $\phi=\phi(v)$ satisfies (6), (7), $\tau(t)=t-\tau_{0}, \sigma(t)=t+\sigma_{0}$, and $\tau_{0}, \sigma_{0} \in[0, \pi / 4)$. We claim that the previous equation is oscillatory provided at least one of the constants $m_{1}, m_{2}$, and $e_{0}$ is large enough. Indeed, it is enough to show that condition (17) is fulfilled by using Corollary 13. It can be done in a similar way as in Example 4 with (20) and Corollary 3. We leave it to the reader.

\section{Auxiliary Results: Qualitative Properties of Concave-Like Functions}

Let $a<b$ be two arbitrary real numbers. It is known that if $x(t)$ is a concave and smooth enough function on $(a, b)$, that is, if $x^{\prime \prime}(t) \leq 0$ for all $t \in(a, b)$, then $x(t)-x(s) \geq x^{\prime}(t)(t-s)$ for all $s, t \in(a, b), s<t$. Moreover, if $x(t)>0$ for all $t \in(a, b)$, then from previous inequality we obtain

$$
\frac{x^{\prime}(t)}{x(t)} \leq \frac{1}{t-s} \quad \forall s, t \in(a, b), s<t .
$$

However, often we do not have any information about the sign of the second-order linear differential operator in (1) except only in a particular case when $r(t) \equiv 1$ and $\phi(v) \equiv v$. Hence the main goal of this section is to find some sufficient conditions on $r(t)$ and $\phi(v)$ such that the assumption " $\left(r(t) \phi\left(x^{\prime}(t)\right)\right)^{\prime} \leq 0$ for all $t \in(a, b)$ " implies the desired inequality (43). It is done in the following results.

Lemma 15. Let $\phi=\phi(v)$ satisfy (6). Let $0<r(s) \leq r(t)$ for all $s, t \in(a, b), s \leq t$. For any function $x \in C^{2}((a, b), \mathbb{R}) \cap$ $C([a, b), \mathbb{R})$ such that $x(t)>0$ for all $t \in(a, b)$, the following statement holds:

$$
\begin{aligned}
& \text { if }\left(r(t) \phi\left(x^{\prime}(t)\right)\right)^{\prime} \leq 0 \quad \forall t \in(a, b), \\
& \text { then } \frac{x^{\prime}(t)}{x(t)} \leq \frac{1}{t-a} \quad \forall t \in(a, b) .
\end{aligned}
$$

Proof. From assumption $\left(r(t) \phi\left(x^{\prime}(t)\right)\right)^{\prime} \leq 0$ for all $t \in(a, b)$, it follows that

$$
r(t) \phi\left(x^{\prime}(t)\right) \leq r(\xi) \phi\left(x^{\prime}(\xi)\right) \quad \forall \xi, t \in(a, b), \quad \xi \leq t .
$$

To the end of this proof, let $t \in(a, b)$ be fixed. Thanks to (6), if $\phi\left(x^{\prime}(t)\right) \leq 0$, then $x^{\prime}(t) \leq 0$ too, because $\phi$ is odd and increasing. In this case, since $r(s)>0$ and $x(s)>0$ on $(a, b)$, it implies that

$$
\frac{x^{\prime}(t)}{x(t)} \leq 0<\frac{1}{t-a} \quad \forall t \in(a, b) .
$$

Thus, it remains to show (44) for the case of $\phi\left(x^{\prime}(t)\right) \geq 0$. From $0<r(\xi) \leq r(t)$ for all $\xi \in(a, b), \xi \leq t$, we obtain $r(t) / r(\xi) \geq 1$ which together with $\phi\left(x^{\prime}(t)\right) \geq 0$ and (45) gives

$$
\phi\left(x^{\prime}(t)\right) \leq \frac{r(t)}{r(\xi)} \phi\left(x^{\prime}(t)\right) \leq \phi\left(x^{\prime}(\xi)\right) \quad \forall \xi \in(a, b), \xi \leq t .
$$


Acting on (47) with $\phi^{-1}$ and using that $\phi^{-1}$ is an increasing function because of (6), we obtain

$$
x^{\prime}(\xi) \geq x^{\prime}(t) \quad \forall \xi \in(a, b), \xi \leq t .
$$

Next, since $x(t)>0$ on $(a, b)$ and $x \in C([a, b), \mathbb{R})$, we have $x(a) \geq 0$. From the mean-value theorem for $x(t)$ on $(a, t)$, we get a $\xi \in(a, t)$ depending on $a$ and $t$ such that $x(t)-x(a)=x^{\prime}(\xi)(t-a)$, and since $x(a) \geq 0$, from (48) we obtain

$$
x(t) \geq x^{\prime}(\xi)(t-a) \geq x^{\prime}(t)(t-a),
$$

which proves the desired inequality in (44).

In the advanced case of (1), that is, when $h_{i}(t)=\sigma_{i}(t) \geq t$, we have the analogous result to Lemma 15.

Lemma 16. Let $\phi=\phi(v)$ satisfy (6). Let $r(s) \geq r(t)>0$ for all $s, t \in(a, b), s \leq t$. For any function $x \in C^{2}((a, b), \mathbb{R}) \cap$ $C((a, b], \mathbb{R})$ such that $x(s)>0$ for all $s \in(a, b)$, the following statement holds:

$$
\begin{aligned}
& \text { if }\left(r(s) \phi\left(x^{\prime}(s)\right)\right)^{\prime} \leq 0 \quad \forall s \in(a, b), \\
& \text { then } \frac{x^{\prime}(s)}{x(s)} \geq-\frac{1}{b-s} \quad \forall s \in(a, b) .
\end{aligned}
$$

Proof. From assumption $\left(r(s) \phi\left(x^{\prime}(s)\right)\right)^{\prime} \leq 0$ for all $s \in(a, b)$, we have

$$
r(s) \phi\left(x^{\prime}(s)\right) \geq r(\xi) \phi\left(x^{\prime}(\xi)\right) \quad \forall s, \xi \in(a, b), s \leq \xi .
$$

To the end of this proof, let $s \in(a, b)$ be fixed. If $\phi\left(x^{\prime}(s)\right) \geq$ 0 , then $x^{\prime}(s) \geq 0$ because $\phi$ is odd and increasing, and since by assumption $x(t)>0$ for all $t \in(a, b)$, we have that

$$
\frac{x^{\prime}(s)}{x(s)} \geq 0 \geq-\frac{1}{b-s} \quad \forall s \in(a, b),
$$

which proves $(50)$ in this case. Let now $\phi\left(x^{\prime}(s)\right) \leq 0$. It implies that $x^{\prime}(s) \leq 0$ and $\phi\left(x^{\prime}(\xi)\right) \leq 0$ because of (51) and $r(s)>0$ for all $s \in(a, b)$. Hence, from $r(\xi) \leq r(s)$ for all $\xi \in(a, b), \xi \geq s$, from (51), and $\phi\left(x^{\prime}(\xi)\right) \leq 0$, we especially conclude that

$$
\phi\left(x^{\prime}(s)\right) \geq \frac{r(\xi)}{r(s)} \phi\left(x^{\prime}(\xi)\right) \geq \phi\left(x^{\prime}(\xi)\right) \quad \forall \xi \in(a, b), \xi \geq s .
$$

Acting on (53) with $\phi^{-1}$ we obtain

$$
\begin{array}{r}
x^{\prime}(s)=\phi^{-1}\left(\phi\left(x^{\prime}(s)\right)\right) \geq \phi^{-1}\left(\phi\left(x^{\prime}(\xi)\right)\right)=x^{\prime}(\xi) \\
\forall \xi \in(a, b), \xi \geq s .
\end{array}
$$

By the Lagrange's mean-value theorem, there exists a $\xi \in$ $(s, b)$ depending on $s, b$ such that $x(b)-x(s)=x^{\prime}(\xi)(b-s)$. Since $x(s)>0$ for all $s \in(a, b)$ and $x \in C((a, b], \mathbb{R})$, from (54) we obtain

$$
-x(s) \leq x(b)-x(s)=x^{\prime}(\xi)(b-s) \leq x^{\prime}(s)(b-s),
$$

which proves the desired inequality. form.

Statement (44) will be frequently used in the following

Corollary 17. Let $\phi=\phi(v)$ satisfy $(6), \tau(t) \leq t$, and $0<$ $r(s) \leq r(t)$ for all $s, t, t_{0} \leq s \leq t$. Let $a_{1}, b_{1}$ be two arbitrary real numbers such that $t_{0} \leq \tau\left(a_{1}\right) \leq a_{1}<b_{1}$. Then for any function $x \in C^{2}\left(\left(\tau\left(a_{1}\right), b_{1}\right), \mathbb{R}\right) \cap C\left(\left[\tau\left(a_{1}\right), b_{1}\right), \mathbb{R}\right)$ such that $x(t)>0$ for all $t \in\left(\tau\left(a_{1}\right), b_{1}\right)$, the following statement holds:

$$
\begin{gathered}
\text { if }\left(r(t) \phi\left(x^{\prime}(t)\right)\right)^{\prime} \leq 0, \quad \forall t \in\left(\tau\left(a_{1}\right), b_{1}\right), \\
\text { then } \frac{x(\tau(t))}{x(t)} \geq \frac{\tau(t)-\tau\left(a_{1}\right)}{t-\tau\left(a_{1}\right)}, \quad \forall t \in\left(a_{1}, b_{1}\right) .
\end{gathered}
$$

Proof. By assumptions of this corollary, we have $\tau(t) \leq t$, $x(t)>0$, and $\left(r(t) \phi\left(x^{\prime}(t)\right)\right)^{\prime} \leq 0$ for all $t \in\left(\tau\left(a_{1}\right), b_{1}\right)$. In particular from statement (44) applied on $(a, b)=\left(\tau\left(a_{1}\right), b_{1}\right)$, we get

$$
\frac{x^{\prime}(t)}{x(t)} \leq \frac{1}{t-\tau\left(a_{1}\right)} \quad \forall t \in\left(\tau\left(a_{1}\right), b_{1}\right) .
$$

Integrating this inequality over the interval $[\tau(t), t]$ for all $t \in\left(a_{1}, b_{1}\right)$, we obtain

$$
\frac{x(t)}{x(\tau(t))} \leq \frac{t-\tau\left(a_{1}\right)}{\tau(t)-\tau\left(a_{1}\right)} \quad \forall t \in\left(a_{1}, b_{1}\right),
$$

which proves the desired inequality in (56).

Statement (50) will appear in the following form.

Corollary 18. Let $a_{1}<b_{1}$ be two arbitrary real numbers. Let $\phi=\phi(v)$ satisfy $(6), \sigma(t) \geq t, r(t)>0$, and $r(s) \geq r(t)$ for all $s, t, s \leq t$. Then for any function $x \in C^{2}\left(\left(a_{1}, \sigma\left(b_{1}\right)\right), \mathbb{R}\right) \cap$ $C\left(\left(a_{1}, \sigma\left(b_{1}\right)\right], \mathbb{R}\right)$ such that $x(t)>0$ for all $t \in\left(a_{1}, \sigma\left(b_{1}\right)\right)$, the following statement holds:

$$
\begin{gathered}
\text { if }\left(r(s) \phi\left(x^{\prime}(s)\right)\right)^{\prime} \leq 0 \quad \forall s \in\left(a_{1}, \sigma\left(b_{1}\right)\right) \\
\text { then } \frac{x(\sigma(s))}{x(s)} \geq \frac{\sigma\left(b_{1}\right)-\sigma(s)}{\sigma\left(b_{1}\right)-s} \quad \forall s \in\left(a_{1}, b_{1}\right) .
\end{gathered}
$$

Proof. Analogously with the proof of Corollary 17, we just need to use the second inequality in $(50)$ on $(a, b)=$ $\left(a_{1}, \sigma\left(b_{1}\right)\right)$ and integrate it over the interval $[s, \sigma(s)]$ for all $s \in\left(a_{1}, b_{1}\right)$.

Next, by Corollary 17 and the arithmetic-geometric mean inequality

$$
\sum_{i=0}^{n} \eta_{i} u_{i} \geq \prod_{i=0}^{n} u_{i}^{\eta_{i}}, \quad u_{i} \geq 0, \quad \eta_{i}>0
$$

we can prove the following proposition.

Proposition 19 (with delay arguments). Let $0<r(s) \leq r(t)$ for all $s, t$ such that $t_{0} \leq s<t$ and let $\phi=\phi(v)$ satisfy (6). Let exponents $\left\{\alpha_{i}\right\}$ and the $(n+1)$-tuple $\left(\eta_{0}, \eta_{1}, \ldots, \eta_{n}\right)$ 
satisfy (10). Let $\tau_{i}(t) \leq t$ on $\left[t_{0}, \infty\right), i \in\{1, \ldots, n\}$, and $\tau_{\min }(t)=\min \left\{\tau_{1}(t), \ldots, \tau_{n}(t)\right\}$. Let $e(t) \leq 0$ and $q_{i}(t) \geq$ 0 on $\left[a_{1}, b_{1}\right]$, where $t_{0} \leq a_{1}<b_{1}$. Then for any function $x \in$ $C^{2}\left(\left(\tau_{\min }\left(a_{1}\right), b_{1}\right), \mathbb{R}\right) \cap C\left(\left[\tau_{\min }\left(a_{1}\right), b_{1}\right), \mathbb{R}\right)$ such that $x(t)>0$ and $\left(r(t) \phi\left(x^{\prime}(t)\right)\right)^{\prime} \leq 0$ for all $t \in\left(\tau_{\min }\left(a_{1}\right), b_{1}\right)$, we have

$$
\begin{array}{r}
\frac{1}{x^{p}(t)}\left[\sum_{i=1}^{n} q_{i}(t)\left(x\left(\tau_{i}(t)\right)\right)^{\alpha_{i}}-e(t)\right] \\
\geq\left(\eta_{0}^{-1}|e(t)|\right)^{\eta_{0}} \prod_{i=1}^{n}\left(\eta_{i}^{-1} q_{i}(t)\right)^{\eta_{i}} \prod_{i=1}^{n}\left(\frac{\tau_{i}(t)-\tau_{i}\left(a_{1}\right)}{t-\tau_{i}\left(a_{1}\right)}\right)^{\alpha_{i} \eta_{i}}, \\
t \in\left(a_{1}, b_{1}\right) .
\end{array}
$$

Proof. By inequality (60) in particular for $u_{i}=\eta_{i}^{-1} q_{i}(t)$ $\left(x\left(\tau_{i}(t)\right)\right)^{\alpha_{i}}$ and $u_{0}=\eta_{0}^{-1} e(t)$, together with (56), we obtain

$$
\begin{aligned}
& \frac{1}{x^{p}(t)}\left[\sum_{i=1}^{n} q_{i}(t)\left(x\left(\tau_{i}(t)\right)\right)^{\alpha_{i}}-e(t)\right] \\
& =\frac{1}{x^{p}(t)}\left[\sum_{i=1}^{n} \eta_{i}\left(\eta_{i}^{-1} q_{i}(t)\left(x\left(\tau_{i}(t)\right)\right)^{\alpha_{i}}\right)+\eta_{0}\left(\eta_{0}^{-1}|e(t)|\right)\right] \\
& \geq \frac{1}{x^{p}(t)} \prod_{i=1}^{n}\left(\eta_{i}^{-1} q_{i}(t)\left(x\left(\tau_{i}(t)\right)\right)^{\alpha_{i}}\right)^{\eta_{i}}\left(\eta_{0}^{-1}|e(t)|\right)^{\eta_{0}} \\
& =\left(\eta_{0}^{-1}|e(t)|\right)^{\eta_{0}} \prod_{i=1}^{n}\left(\eta_{i}^{-1} q_{i}(t)\right)^{\eta_{i}} \frac{\prod_{i=1}^{n}\left(x\left(\tau_{i}(t)\right)\right)^{\alpha_{i} \eta_{i}}}{(x(t))^{\sum_{i=1}^{n} \alpha_{i} \eta_{i}}} \\
& =\left(\eta_{0}^{-1}|e(t)|\right)^{\eta_{0}} \prod_{i=1}^{n}\left(\eta_{i}^{-1} q_{i}(t)\right)^{\eta_{i}} \frac{\prod_{i=1}^{n}\left(x\left(\tau_{i}(t)\right)\right)^{\alpha_{i} \eta_{i}}}{\prod_{i=1}^{n}(x(t))^{\alpha_{i} \eta_{i}}} \\
& =\left(\eta_{0}^{-1}|e(t)|\right)^{\eta_{0}} \prod_{i=1}^{n}\left(\eta_{i}^{-1} q_{i}(t)\right)^{\eta_{i}} \prod_{i=1}^{n}\left(\frac{x\left(\tau_{i}(t)\right)}{x(t)}\right)^{\alpha_{i} \eta_{i}} \\
& \geq\left(\eta_{0}^{-1}|e(t)|\right)^{\eta_{0}} \prod_{i=1}^{n}\left(\eta_{i}^{-1} q_{i}(t)\right)^{\eta_{i}} \prod_{i=1}^{n}\left(\frac{\tau_{i}(t)-\tau_{i}\left(a_{1}\right)}{t-\tau_{i}\left(a_{1}\right)}\right)^{\alpha_{i} \eta_{i}}
\end{aligned}
$$

which proves this proposition.

Proposition 20 (with advanced arguments). Let $r(s) \geq r(t)>$ 0 for all $s, t$ such that $t_{0} \leq s<t$ and let $\phi=\phi(v)$ satisfy (6). Let exponents $\left\{\alpha_{i}\right\}$ and the $(n+1)$-tuple $\left(\eta_{0}, \eta_{1}, \ldots, \eta_{n}\right)$ satisfy (10). Let $\sigma_{i}(t) \geq t$ on $\left[t_{0}, \infty\right), i \in\{1, \ldots, n\}$, and $\sigma_{\max }(t)=\max \left\{\sigma_{1}(t), \ldots, \sigma_{n}(t)\right\}$. Let $e(t) \leq 0$ and $q_{i}(t) \geq 0$ on $\left[a_{1}, b_{1}\right]$, where $t_{0} \leq a_{1}<b_{1}$. Then for any function $x \in$
$C^{2}\left(\left(a_{1}, \sigma_{\max }\left(b_{1}\right)\right), \mathbb{R}\right) \cap C\left(\left(a_{1}, \sigma_{\max }\left(b_{1}\right)\right], \mathbb{R}\right)$ such that $x(t)>0$ and $\left(r(t) \phi\left(x^{\prime}(t)\right)\right)^{\prime} \leq 0$ for all $t \in\left(a_{1}, \sigma_{\max }\left(b_{1}\right)\right)$, we have

$$
\begin{array}{r}
\frac{1}{x^{p}(t)}\left[\sum_{i=1}^{n} q_{i}(t)\left(x\left(\sigma_{i}(t)\right)\right)^{\alpha_{i}}-e(t)\right] \\
\geq\left(\eta_{0}^{-1}|e(t)|\right)^{\eta_{0}} \prod_{i=1}^{n}\left(\eta_{i}^{-1} q_{i}(t)\right)^{\eta_{i}} \prod_{i=1}^{n}\left(\frac{\sigma_{i}\left(b_{1}\right)-\sigma_{i}(t)}{\sigma_{i}\left(b_{1}\right)-t}\right)^{\alpha_{i} \eta_{i}}, \\
t \in\left(a_{1}, b_{1}\right) .
\end{array}
$$

Proof. It is very similar to the proof of Proposition 19 but instead of Corollary 17 we need to use Corollary 18.

According to Corollary 17, we are able to prove the next useful proposition in which we also use the well-known Young inequality $X, Y \geq 0$ :

$$
X^{\gamma}+(\gamma-1) Y^{\gamma} \geq \gamma X Y^{\gamma-1}, \quad \gamma>1 \text {. }
$$

Proposition 21 (with delay-advanced arguments). Let $r(t) \equiv$ const $>0$ on $\left[t_{0}, \infty\right)$, real numbers $\alpha_{i}>p>0$ for all $i=1,2, \ldots, n$ and $m \in \mathbb{N}, 1<m<n$. Let $\phi=\phi(v)$ satisfy $(6)$, $\tau_{i}(t) \leq t, i \in\{1, \ldots, m\}$ and $\sigma_{i}(t) \geq t, i \in\{m+1, \ldots, n\}$ on $\left[t_{0}, \infty\right)$, and $\tau_{\min }(t)=\min \left\{\tau_{1}(t), \ldots, \tau_{m}(t)\right\}, \sigma_{\max }(t)=$ $\max \left\{\sigma_{m+1}(t), \ldots, \sigma_{n}(t)\right\}$. Let $e(t) \leq 0$ and $q_{i}(t) \geq 0$ on $\left[a_{1}, b_{1}\right]$, where $t_{0} \leq a_{1}<b_{1}$. Then for any function $x \in$ $C^{2}\left(\left(\tau_{\min }\left(a_{1}\right), \sigma_{\max }\left(b_{1}\right)\right), \mathbb{R}\right) \cap C\left(\left[\tau_{\min }\left(a_{1}\right), \sigma_{\max }\left(b_{1}\right)\right], \mathbb{R}\right)$ such that $x(t)>0$ and $\left(r(t) \phi\left(x^{\prime}(t)\right)\right)^{\prime} \leq 0$ on $\left(\tau_{\min }\left(a_{1}\right), \sigma_{\max }\left(b_{1}\right)\right)$, we have

$$
\begin{aligned}
\frac{1}{x^{p}(t)}\left[\sum_{i=1}^{m} q_{i}(t)\left(x\left(\tau_{i}(t)\right)\right)^{\alpha_{i}}-\frac{1}{2} e(t)\right] \\
+\frac{1}{x^{p}(t)}\left[\sum_{i=m+1}^{n} q_{i}(t)\left(x\left(\sigma_{i}(t)\right)\right)^{\alpha_{i}}-\frac{1}{2} e(t)\right] \\
\geq \sum_{i=1}^{m} \frac{\alpha_{i}}{p} q_{i}^{p / \alpha_{i}}(t)\left(\frac{p|e(t)|}{2\left(\alpha_{i}-p\right) m}\right)^{\left(\alpha_{i}-p\right) / \alpha_{i}} \\
\left.\quad \times\left(\frac{\tau_{i}(t)-\tau_{i}\left(a_{1}\right)}{t-\tau_{i}\left(a_{1}\right)}\right)^{p}\right)^{\left(\alpha_{i}-p\right) / \alpha_{i}} \\
+\sum_{i=m+1}^{n} \frac{\alpha_{i}}{p} q_{i}^{p / \alpha_{i}}(t)\left(\frac{p(t) \mid}{2\left(\alpha_{i}-p\right)(n-m-1)}\right) \\
\quad \times\left(\frac{\sigma_{i}\left(b_{1}\right)-\sigma_{i}(t)}{\sigma_{i}\left(b_{1}\right)-t}\right)^{p},
\end{aligned}
$$

for all $t \in\left(a_{1}, b_{1}\right)$.

Proof. First of all, by inequality (64) in particular for $\gamma=$ $\alpha_{i} / p$ and

$$
X=\left(q_{i}(t)\right)^{p / \alpha_{i}} x^{p}\left(\tau_{i}(t)\right), \quad Y=\left(\frac{p|e(t)|}{2\left(\alpha_{i}-p\right) m}\right)^{p / \alpha_{i}}
$$


together with (56), we obtain

$$
\begin{aligned}
& \frac{1}{x^{p}(t)}\left[\sum_{i=1}^{m} q_{i}(t)\left(x\left(\tau_{i}(t)\right)\right)^{\alpha_{i}}-\frac{1}{2} e(t)\right] \\
& =\frac{1}{x^{p}(t)} \sum_{i=1}^{m}\left\{\left[\left(q_{i}(t)\right)^{p / \alpha_{i}} x^{p}\left(\tau_{i}(t)\right)\right]^{\alpha_{i} / p}\right. \\
& \left.+\left(\frac{\alpha_{i}}{p}-1\right)\left[\left(\frac{p|e(t)|}{2\left(\alpha_{i}-p\right) m}\right)^{p / \alpha_{i}}\right]^{\alpha_{i} / p}\right\} \\
& \geq \frac{1}{x^{p}(t)} \sum_{i=1}^{m} \frac{\alpha_{i}}{p}\left(q_{i}(t)\right)^{p / \alpha_{i}}\left(\frac{p|e(t)|}{2\left(\alpha_{i}-p\right) m}\right)^{\left(\alpha_{i}-p\right) / \alpha_{i}} x^{p}\left(\tau_{i}(t)\right) \\
& =\sum_{i=1}^{m} \frac{\alpha_{i}}{p}\left(q_{i}(t)\right)^{p / \alpha_{i}}\left(\frac{p|e(t)|}{2\left(\alpha_{i}-p\right) m}\right)^{\left(\alpha_{i}-p\right) / \alpha_{i}}\left[\frac{x\left(\tau_{i}(t)\right)}{x(t)}\right]^{p} \\
& \geq \sum_{i=1}^{m} \frac{\alpha_{i}}{p}\left(q_{i}(t)\right)^{p / \alpha_{i}}\left(\frac{p|e(t)|}{2\left(\alpha_{i}-p\right) m}\right)^{\left(\alpha_{i}-p\right) / \alpha_{i}} \\
& \times\left(\frac{\tau_{i}(t)-\tau_{i}\left(a_{1}\right)}{t-\tau_{i}\left(a_{1}\right)}\right)^{p} .
\end{aligned}
$$

In the same way, one can show that

$$
\begin{gathered}
\frac{1}{x^{p}(t)}\left[\sum_{i=m+1}^{n} q_{i}(t)\left(x\left(\sigma_{i}(t)\right)\right)^{\alpha_{i}}-\frac{1}{2} e(t)\right] \\
\geq \sum_{i=m+1}^{n} \frac{\alpha_{i}}{p} q_{i}^{p / \alpha_{i}}(t)\left(\frac{p|e(t)|}{2\left(\alpha_{i}-p\right)(n-m-1)}\right)^{\left(\alpha_{i}-p\right) / \alpha_{i}} \\
\times\left(\frac{\sigma_{i}\left(b_{1}\right)-\sigma_{i}(t)}{\sigma_{i}\left(b_{1}\right)-t}\right)^{p} .
\end{gathered}
$$

The previous two inequalities prove this proposition.

\section{Proof of Main Results}

Proof of Theorem 1. If the assertion of this theorem does not hold, then there is a nonoscillatory solution $x(t)$ of (1) such that $x(t) \neq 0$ for all $t \geq T$ and some $T \geq t_{0}$. Moreover, it is enough to work only with the case $x(t)>0$ and $e(t) \leq 0$ on $\left[\tau_{\min }\left(a_{1}\right), b_{1}\right]$, since the second case $x(t)<0$ and $-e(t) \leq 0$ on $\left[\tau_{\min }\left(a_{2}\right), b_{2}\right]$ can be transformed into the first one. Indeed, multiplying equation (1) by -1 and using assumptions that $\phi(v)$ and $f(u)$ are odd functions, we have that (1) is equivalent to

$$
\begin{aligned}
(r(t) & \left.\phi\left(-x^{\prime}(t)\right)\right)^{\prime}+\sum_{i=1}^{n} r_{i}(t) f\left(-x\left(\tau_{i}(t)\right)\right) \\
& +\sum_{i=1}^{n} q_{i}(t)\left|\left(-x\left(\tau_{i}(t)\right)\right)\right|^{\alpha_{i}} \operatorname{sgn}\left(-x\left(\tau_{i}(t)\right)\right)=-e(t),
\end{aligned}
$$

The proof is outlined in the following three steps.

Step 1. Next, for any $\lambda_{1}>0$ the following function is well defined:

$$
\bar{\omega}_{1}(t)=-\frac{\lambda_{1} r(t) \phi\left(x^{\prime}(t)\right)}{x^{p}(t)}, \quad t \in\left[a_{1}, b_{1}\right],
$$

and $\bar{\omega}_{1} \in C^{1}\left(\left[a_{1}, b_{1}\right), \mathbb{R}\right)$. Since $\lambda_{1}>0$ and $r(t)>0$ on $\left[a_{1}, b_{1}\right]$, from the previous equality we get

$$
\begin{gathered}
\left|\phi\left(x^{\prime}(t)\right)\right|=\frac{\left|\bar{\omega}_{1}(t)\right|}{\lambda_{1} r(t)} x^{p}(t), \\
\bar{\omega}_{1}^{\prime}(t)=-\frac{\lambda_{1}\left(r(t) \phi\left(x^{\prime}(t)\right)\right)^{\prime}}{x^{p}(t)}+\frac{p \lambda_{1} r(t) \phi\left(x^{\prime}(t)\right)}{x^{p+1}(t)} x^{\prime}(t) .
\end{gathered}
$$

Using (1) and assumptions (7) and (9), from the second equality of (71), we get

$$
\begin{aligned}
& \frac{d}{d t} \bar{\omega}_{1}(t) \geq \frac{p}{\left(\lambda_{1} r(t)\right)^{1 / p}}\left|\bar{\omega}_{1}(t)\right|^{(p+1) / p} \\
& +\lambda_{1} K \sum_{i=1}^{n} r_{i}(t)\left(\frac{x\left(\tau_{i}(t)\right)}{x(t)}\right)^{p} \\
& +\frac{\lambda_{1}}{x^{p}(t)}\left[\sum_{i=1}^{n} q_{i}(t)\left(x\left(\tau_{i}(t)\right)\right)^{\alpha_{i}}-e(t)\right],
\end{aligned}
$$

where $t \in\left(a_{1}, b_{1}\right)$. Next, using assumptions (12) and (13) in (1) for all $t \in\left(\tau_{\min }\left(a_{1}\right), b_{1}\right)$ we easily conclude that $x(t)$ satisfies all assumptions of Corollary 17 and Proposition 19. Hence from (56), (61), and (72) we observe

$$
\begin{array}{r}
\frac{d}{d t} \bar{\omega}_{1}(t) \geq \frac{p}{\left(\lambda_{1} r(t)\right)^{1 / p}}\left|\bar{\omega}_{1}(t)\right|^{(p+1) / p}+\lambda_{1}\left(R_{1}(t)+Q_{1}(t)\right), \\
t \in\left(a_{1}, b_{1}\right) .
\end{array}
$$

Step 2. In this step, we need the next elementary proposition.

Proposition 22. Let $p>0$ and let $\pi_{p}$ be a positive number defined by

$$
\pi_{p}=\frac{\pi}{((p+1) / p) \sin (p \pi /(p+1))} .
$$

Then there is an increasing odd function $y=y(s), y \in$ $C^{1}\left(\left(-\pi_{p}, \pi_{p}\right), \mathbb{R}\right)$, such that

$$
\begin{gathered}
y^{\prime}(s)=1+|y(s)|^{(p+1) / p}, \quad s \in\left(-\pi_{p}, \pi_{p}\right), \\
y(0)=0, \quad y\left(\pi_{p}\right)=\infty .
\end{gathered}
$$

Moreover, for $p=1$ we have $\pi_{p}=\pi / 2$ and we can take $y(s)=\tan (s), s \in(-\pi / 2, \pi / 2)$. 
Proof. Let $z=z(t)$ be a function defined by

$$
z(t)=\int_{0}^{t} \frac{1}{1+|\tau|^{(p+1) / p}} d \tau, \quad t \in \mathbb{R} .
$$

It is not difficult to see that $z(\infty)=\pi_{p}$ (see [24]) and $z$ is a bijection from $\mathbb{R}$ on interval $(-z(\infty), z(\infty))$ and that the function $y(s)$ determined by the formula $y(s)=$ $z^{-1}(s)$, where $z^{-1}(s)$ is the inverse function of $z(t)$, satisfies all properties given in (75).

Let now $s_{j} \in(-\pi / 2, \pi / 2)$ be such that $y\left(s_{j}\right)=$ $\bar{\omega}_{1}\left(a_{j}\right)$ (such an $s_{j}$ exists since $y(s)$ is a bijection from $(-\pi / 2, \pi / 2)$ to $(-\infty, \infty)), J=\left[a_{1}, b_{1}\right] \cup\left[a_{2}, b_{2}\right]$, and let $C_{0}(t)$ be a function defined by

$$
C_{0}(t)=\frac{1}{2 \pi_{p}} \min \left\{\frac{p}{\left(\lambda_{1} r(t)\right)^{1 / p}}, \lambda_{1}\left(R_{1}(t)+Q_{1}(t)\right)\right\},
$$

Because of (11), we have $c_{0 j}:=\int_{a_{j}}^{b_{j}} C_{0}(\tau) d \tau \geq 1$. Hence,

$$
\begin{array}{r}
\frac{2 \pi_{p}}{c_{0 j}} C_{0}(t) \leq \min \left\{\frac{p}{\left(\lambda_{1} r(t)\right)^{1 / p}}, \lambda_{1}\left(R_{1}(t)+Q_{1}(t)\right)\right\}, \\
t \in J .
\end{array}
$$

Next, let $V_{1}(t)$ and $V_{2}(t)$ be two functions defined by

$$
V_{j}(t)=s_{j}+\frac{2 \pi_{p}}{c_{0 j}} \int_{a_{j}}^{t} C_{0}(\tau) d \tau, \quad t \in\left[a_{j}, b_{j}\right], j \in\{1,2\} .
$$

Since $C_{0}(t) \geq 0$ for all $t \in J$, we have $V_{j}\left(a_{j}\right)=s_{j}<\pi_{p}$ and $V_{j}\left(b_{j}\right)>2 \pi_{p}+s_{j}$. Since $V_{j}(t)$ is continuous, it gives the existence of $T_{j}^{*} \in\left(a_{j}, b_{j}\right)$ such that $V_{j}\left(T_{j}^{*}\right)=\pi_{p}$. Hence, the function $\underline{\omega}_{j}(t)=\tan \left(V_{j}(t)\right), t \in\left(a_{j}, T_{j}^{*}\right)$, satisfies

$$
\begin{gathered}
\underline{\omega}_{j}\left(a_{j}\right)=y\left(V_{j}\left(a_{j}\right)\right)=y\left(s_{j}\right)=\bar{\omega}_{1}\left(a_{j}\right), \\
\underline{\omega}_{j}\left(T_{j}^{*}\right)=y\left(V_{j}\left(T_{j}^{*}\right)\right)=y\left(\pi_{p}\right)=\infty,
\end{gathered}
$$

and because of (78), we obtain

$$
\begin{aligned}
\underline{\omega}_{j}^{\prime}(t)= & y^{\prime}\left(V_{j}(t)\right) V_{j}^{\prime}(t) \\
= & \left(1+\left|y\left(V_{j}(t)\right)\right|^{(p+1) / p}\right) V_{j}^{\prime}(t) \\
= & \left(1+\left|\underline{\omega}_{j}(t)\right|^{(p+1) / p}\right) V_{j}^{\prime}(t) \\
\leq & \left(1+\left|\underline{\omega}_{j}(t)\right|^{(p+1) / p}\right) \frac{2 \pi}{c_{0 j}} C_{0}(t) \\
\leq & \left(1+\left|\underline{\omega}_{j}(t)\right|^{(p+1) / p}\right) \\
& \quad \times \min \left\{\frac{p}{\left(\lambda_{1} r(t)\right)^{1 / p}}, \lambda_{1}\left(R_{1}(t)+Q_{1}(t)\right)\right\},
\end{aligned}
$$

that is

$$
\begin{array}{r}
\underline{\omega}_{j}^{\prime}(t) \leq \frac{p}{\left(\lambda_{1} r(t)\right)^{1 / p}}\left|\underline{\omega}_{j}(t)\right|^{(p+1) / p}+\lambda_{1}\left(R_{1}(t)+Q_{1}(t)\right), \\
t \in\left[a_{j}, T_{j}^{*}\right) .
\end{array}
$$

Step 3. We claim that

$$
\underline{\omega}_{1}(t) \leq \bar{\omega}_{1}(t) \quad \text { on }\left[a_{1}, T_{1}^{*}\right) \text {. }
$$

In order to prove inequality (83), we need the following proposition.

Proposition 23. Let $a<T^{*}$ and $\varphi, \psi \in C^{1}\left(\left[a, T^{*}\right), \mathbb{R}\right)$ be two functions satisfying on $\left[a, T^{*}\right)$, respectively,

$$
\begin{aligned}
& \varphi^{\prime} \leq \frac{p}{\left(\lambda_{1} r(t)\right)^{1 / p}}|\varphi|^{(p+1) / p}+\lambda_{1}\left(R_{1}(t)+Q_{1}(t)\right), \\
& \psi^{\prime} \geq \frac{p}{\left(\lambda_{1} r(t)\right)^{1 / p}}|\psi|^{(p+1) / p}+\lambda_{1}\left(R_{1}(t)+Q_{1}(t)\right) .
\end{aligned}
$$

Then the following statement holds:

$$
\varphi(a) \leq \psi(a) \text { implies } \varphi(t) \leq \psi(t) \quad \forall t \in\left[a, T^{*}\right) .
$$

Proof. If we denote by

$$
F(t, u)=\frac{p}{\left(\lambda_{1} r(t)\right)^{1 / p}} u^{(p+1) / p}+\lambda_{1}\left(R_{1}(t)+Q_{1}(t)\right),
$$

then (84) can be rewritten in the form $\varphi^{\prime} \leq F(t, \varphi)$ and $\psi^{\prime} \geq$ $F(t, \psi)$ on $\left[a, T^{*}\right)$. Also, it is not difficult to check that $F(t, u)$ is locally Lipschitz function in the second variable and bounded in the first variable on bounded interval $\left[a, T^{*}\right)$. Hence, we may apply the pointwise comparison principle from [12, Lemma 19] and, consequently, we conclude that statement (85) holds, which proves this proposition.

Next, we proceed with the proof of inequality (83). By (80) we know that $\underline{\omega}_{j}\left(a_{j}\right)=\bar{\omega}_{1}\left(a_{j}\right)$ which, together with (73) and (82), ensures that we may apply Proposition 23. Now, the desired inequality (83) immediately follows from statement (85).

Next, from the second equality of (80) and inequality (83) we conclude that $\bar{\omega}_{1}\left(T_{1}^{*}\right)=\infty$. It contradicts the fact that $\bar{\omega}_{1} \in C^{1}\left(\left[a_{1}, b_{1}\right)\right)$. Hence, $x(t)$ can not be oscillatory as it is supposed at the beginning of this proof and we conclude that (1) is oscillatory.

Proof of Corollary 2. From (16), we see that $r(t) \equiv$ 1 and $r_{i}(t) \equiv 0$. Next, to the end of this proof, let $\lambda_{j}$ be defined by

$$
\lambda_{j}=\left(\frac{p}{\max _{\left[a_{j}, b_{j}\right]} Q_{j}(t)}\right)^{p /(p+1)}, j \in\{1,2\} .
$$


Now, we can rewrite (17) in the form

$$
\frac{1}{2 \pi_{p}} \int_{a_{j}}^{b_{j}} \lambda_{j} Q_{j}(t) d t \geq 1 .
$$

Since $\max _{\left[a_{j}, b_{j}\right]} Q_{j}(t) \leq Q_{j}(t)$, it is clear that

$$
\frac{p}{\lambda_{j}^{1 / p}} \geq \lambda_{j} Q_{j}(t), \quad t \in\left[a_{j}, b_{j}\right],
$$

and so

$$
\min \left\{\frac{p}{\lambda_{j}^{1 / p}}, \lambda_{j} Q_{j}(t)\right\}=\lambda_{j} Q_{j}(t)
$$

Together with assumption (88) we show that

$$
\begin{aligned}
& \frac{1}{2 \pi_{p}} \int_{a_{j}}^{b_{j}} \min \left\{\frac{p}{\left(\lambda_{j} r(t)\right)^{1 / p}}, \lambda_{j}\left(R_{j}(t)+Q_{j}(t)\right)\right\} d t \\
& \quad=\frac{1}{2 \pi_{p}} \int_{a_{j}}^{b_{j}} \min \left\{\frac{p}{\lambda_{j}^{1 / p}}, \lambda_{j} Q_{j}(t)\right\} d t \\
& \quad=\frac{1}{2 \pi_{p}} \int_{a_{j}}^{b_{j}} \lambda_{j} Q_{j}(t) d t \geq 1 .
\end{aligned}
$$

It proves that desired condition (11) is fulfilled. Thus, this corollary follows from Theorem 1.

Proofs of Theorems 10 and 12. It follows by the same line of arguments as in the proof of Theorem 1, but instead of Proposition 19 we use Propositions 20 and 21, respectively.

\section{Acknowledgment}

This work is supported by the Ministry of Science of the Republic of Croatia under Grant no. 036-0361621-1291. The author has dedicated this work to his professors: Asim Musaefendić-Gymnasium Maglaj; Svetozar Kurepa, Nikola Sarapa, Branko Najman and Darko Žubrinić-graduate and postgraduate studies on Faculty of Sciences University of Zagreb; Jean-Pierre Puel and Jacques-Louis Lionspostdoctoral study in Paris 1996/97.

\section{References}

[1] Y. Bai and L. Liu, "New oscillation criteria for second-order delay differential equations with mixed nonlinearities," Discrete Dynamics in Nature and Society, vol. 2010, Article ID 796256, 9 pages, 2010.

[2] S. Murugadass, E. Thandapani, and S. Pinelas, "Oscillation criteria for forced second-order mixed type quasilinear delay differential equations," Electronic Journal of Differential Equations, vol. 2010, article 73, 9 pages, 2010.

[3] T. S. Hassan, L. Erbe, and A. Peterson, "Forced oscillation of second order differential equations with mixed nonlinearities," Acta Mathematica Scientia B, vol. 31, no. 2, pp. 613-626, 2011.
[4] C. Li and S. Chen, "Oscillation of second-order functional differential equations with mixed nonlinearities and oscillatory potentials," Applied Mathematics and Computation, vol. 210, no. 2, pp. 504-507, 2009.

[5] A. Zafer, "Interval oscillation criteria for second order superhalf linear functional differential equations with delay and advanced arguments," Mathematische Nachrichten, vol. 282, no. 9, pp. 1334-1341, 2009.

[6] A. F. Güvenilir and A. Zafer, "Second-order oscillation of forced functional differential equations with oscillatory potentials," Computers \& Mathematics with Applications, vol. 51, no. 9-10, pp. 1395-1404, 2006.

[7] A. F. Güvenilir, "Interval oscillation of second-order functional differential equations with oscillatory potentials," Nonlinear Analysis: Theory, Methods \& Applications, vol. 71, no. 12, pp. e2849-e2854, 2009.

[8] Q. Kong, "Interval criteria for oscillation of second-order linear ordinary differential equations," Journal of Mathematical Analysis and Applications, vol. 229, no. 1, pp. 258-270, 1999.

[9] Y. G. Sun and J. S. W. Wong, "Oscillation criteria for second order forced ordinary differential equations with mixed nonlinearities," Journal of Mathematical Analysis and Applications, vol. 334, no. 1, pp. 549-560, 2007.

[10] S. Jing, "A new oscillation criterion for forced second-order quasilinear differential equations," Discrete Dynamics in Nature and Society, vol. 2011, Article ID 428976, 8 pages, 2011.

[11] L. H. Erbe, Q. Kong, and B. G. Zhang, Theory for Functional Differential Equations, vol. 190 of Pure and Applied Mathematics, Marcel Dekker, New York, NY, USA, 1994.

[12] M. Pašić, "Fite-Wintner-Leighton-type oscillation criteria for second-order differential equations with nonlinear damping," Abstract and Applied Analysis, vol. 2013, Article ID 852180, 10 pages, 2013.

[13] M. Pašić, "New interval oscillation criteria for forced secondorder differential equations with nonlinear damping," International Journal of Mathematical Analysis, vol. 7, no. 25, pp. 12391255, 2013.

[14] D. Gilbarg and N. S. Trudinger, Elliptic Partial Differential Equations of Second Order, Springer, Berlin, Germany, 2001, Reprint of the 1998 edition.

[15] P. L. de Nápoli and J. P. Pinasco, "A Lyapunov inequality for monotone quasilinear operators," Differential and Integral Equations, vol. 18, no. 10, pp. 1193-1200, 2005.

[16] M. Feng, "Periodic solutions for prescribed mean curvature Liénard equation with a deviating argument," Nonlinear Analysis: Real World Applications, vol. 13, no. 3, pp. 1216-1223, 2012.

[17] I. Rachůnková and M. Tvrdý, "Second-order periodic problem with $\phi$-Laplacian and impulses," Nonlinear Analysis: Theory, Methods and Applications, vol. 63, no. 5-7, pp. e257-e266, 2005.

[18] L. Ferracuti and F. Papalini, "Boundary-value problems for strongly non-linear multivalued equations involving different $\phi$-Laplacians," Advances in Differential Equations, vol. 14, no. 56, pp. 541-566, 2009.

[19] I. Yermachenko and F. Sadyrbaev, "Quasilinearization technique for $\phi$-Laplacian type equations," International Journal of Mathematics and Mathematical Sciences, vol. 2012, Article ID 975760, 11 pages, 2012.

[20] J. Henderson, A. Ouahab, and S. Youcefi, "Existence and topological structure of solution sets for $\phi$-Laplacian impulsive differential equations," Electronic Journal of Differential Equations, vol. 2012, article 56, 16 pages, 2012. 
[21] V. Kolmanovskii and A. Myshkis, Introduction to the Theory and Applications of Functional Differential Equations, vol. 463 of Mathematics and Its Applications, Kluwer Academic, Dordrecht, The Netherlands, 1999.

[22] R. Finn, Equilibrium Capillary Surface, Springer, New York, NY, USA, 1986.

[23] E. Giusti, Minimal Surfaces and Functions of Bounded Variations, Birkhäuser, Basel, Switzerland, 1984.

[24] I. N. Bronshtein, K. A. Semendyayev, G. Musiol, and H. Muehlig, Handbook of Mathematics, Springer, Berlin, Germany, 2007. 


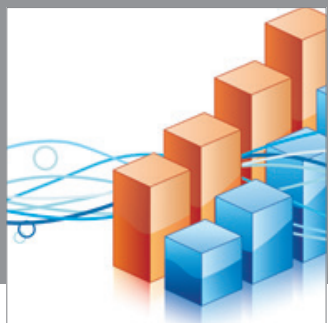

Advances in

Operations Research

mansans

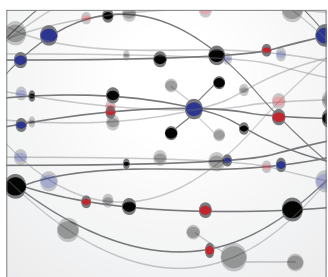

The Scientific World Journal
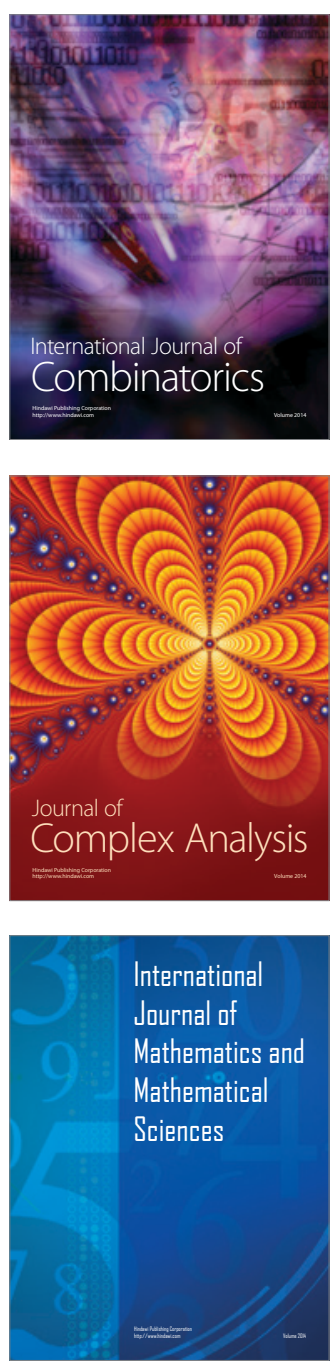
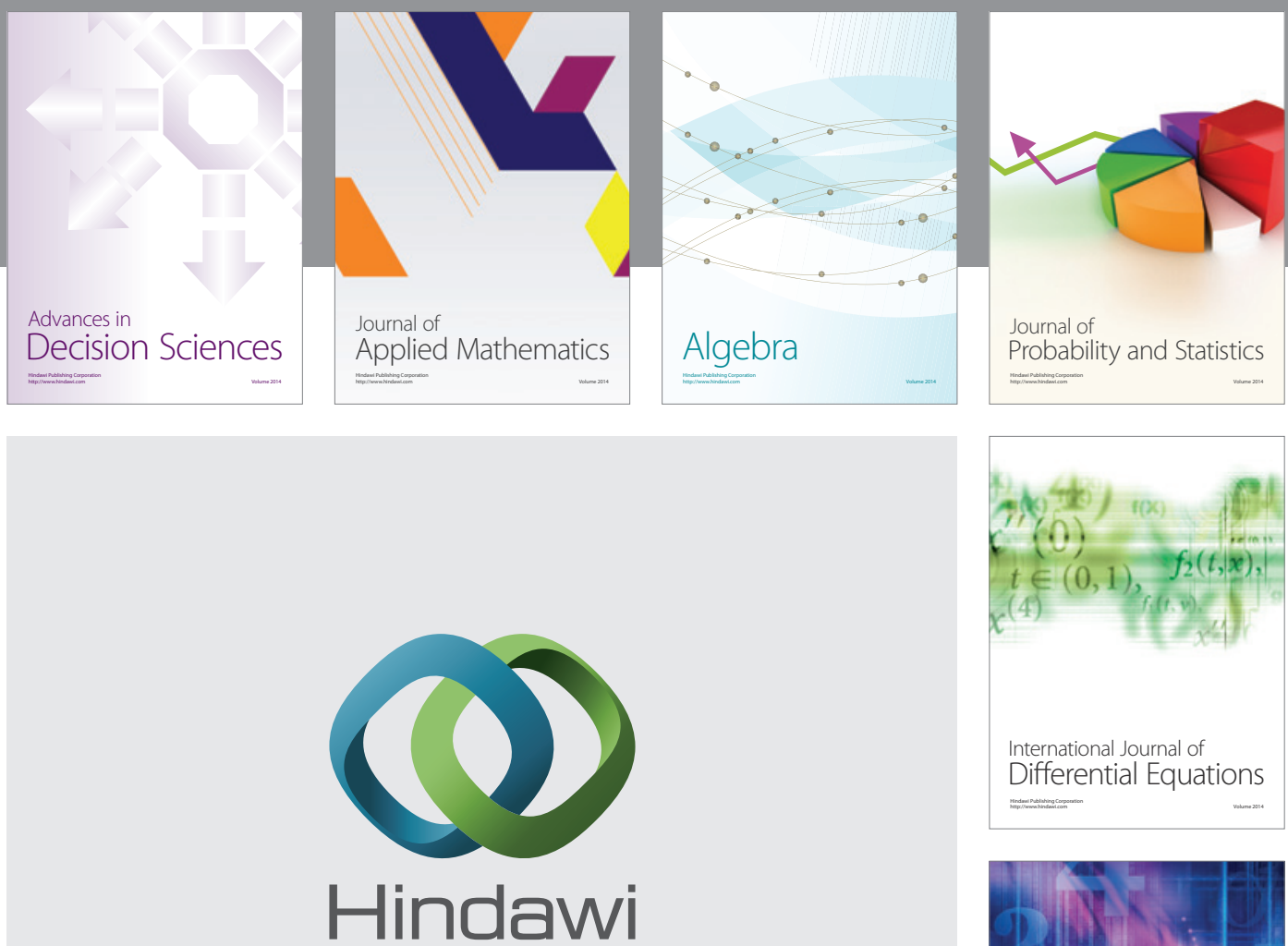

Submit your manuscripts at http://www.hindawi.com
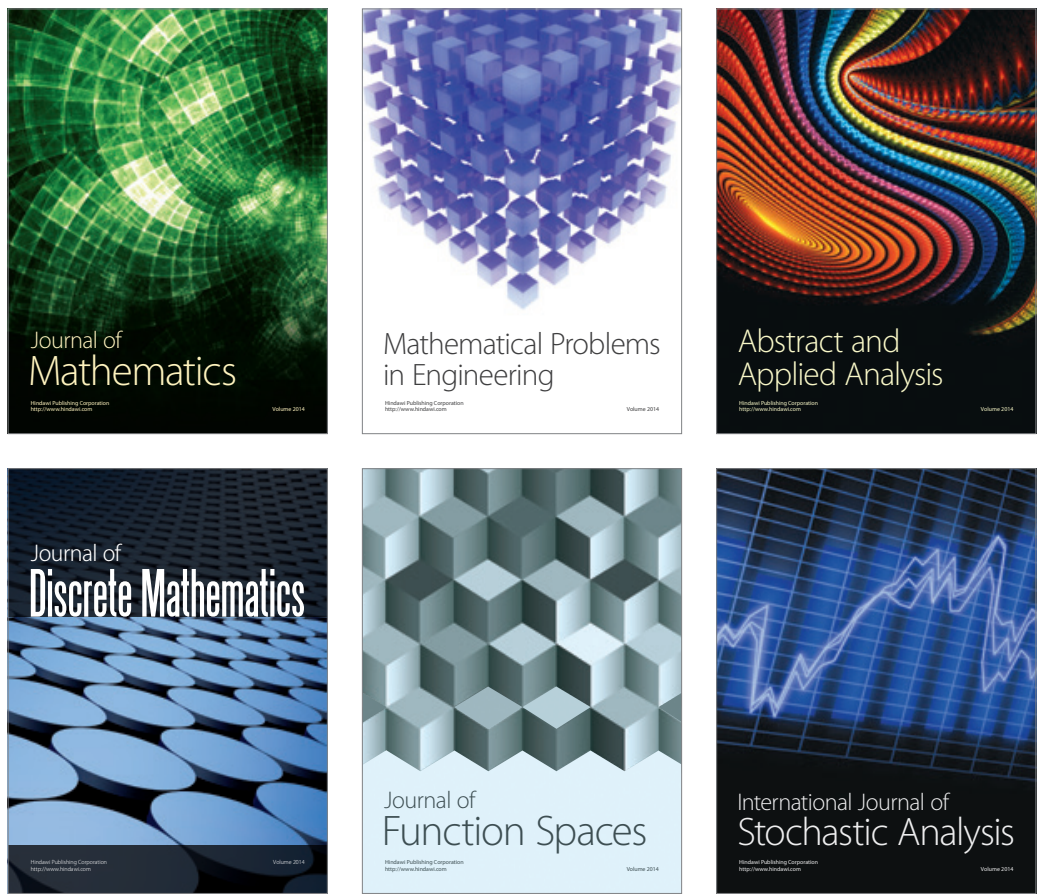

Journal of

Function Spaces

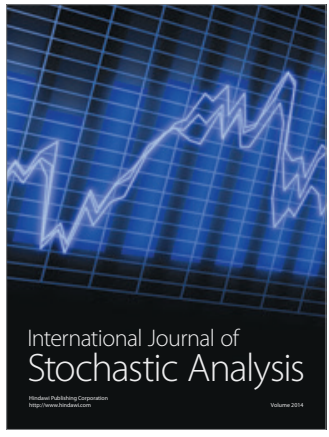

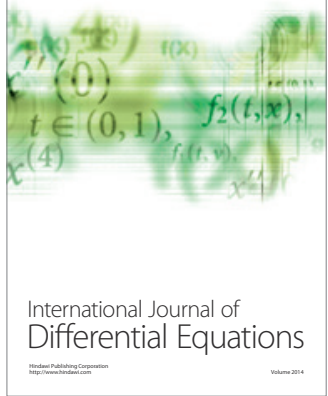
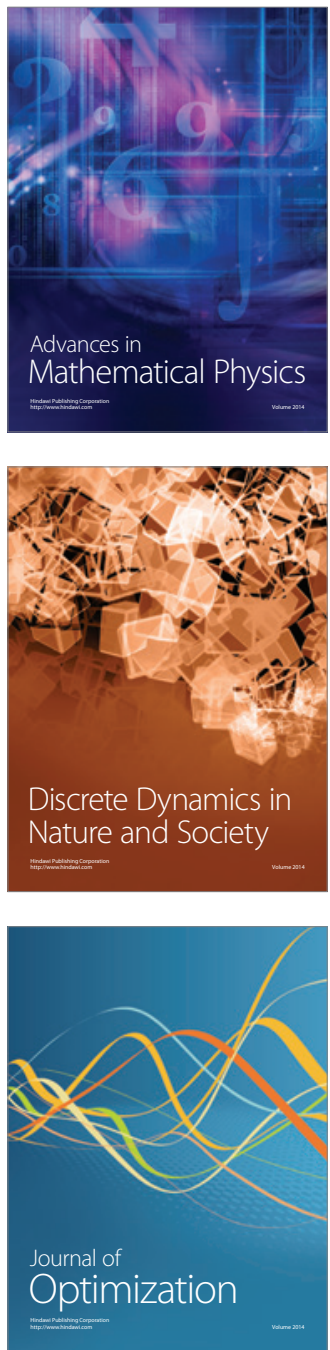